\title{
Comparatative Analysis of Energy Detection Spectrum Sensing of Cognitive Radio Under Wireless Environment Using SEAMCAT
}

\author{
A.S.Kang ${ }^{1}$, Renu Vig ${ }^{2}$, Jasvir Singh ${ }^{3}$, Jaisukh Paul Singh ${ }^{4}$ \\ Deptt.of Electronics and Communication ${ }^{1,2}$ \\ UIET, Panjab University, Chandigarh, India \\ Deptt of Electronics Technology, \\ Guru Nanak Dev University, Amritsar, India
}

\begin{abstract}
In the recent years, the Cognitive Radio technology imposed itself as a good solution to enhance the utilization of unused spectrum and globalized the radio environment for different band users that utilize or require different techniques for transmission. In this paper, the energy detection spectrum sensing technique that is used to detect the presence of unknown deterministic signal is studied under the non-time dispersive fading environment using the Hata propagation model for picocell communication systems. The different aspects of nontime dispersive fading regions over energy detection spectrum sensing and impact of changing a detection threshold of the secondary user Cognitive Radio on interference at primary user for non-cooperative spectrum access have been studied in the terms of probability of interference. The entire Comparatative Analysis of Spectrum Sensing in Cognitive radio has been carried out with the aid of SEAMCAT software platform.
\end{abstract}

Keywords-Cognitive Radio; Primary User; Secondary User; Detection Threshold; Interference Probability;Energy Detection; Desired/interfering/sensing received signal strength

\section{INTRODUCTION}

Cognitive radio is a futuristic technology that will delimit the congestion problem in a radio spectrum, and globalize the radio environment for different band users which use different techniques for transmission. In today's wireless communication scenario, the Radio Frequency spectrum is occupied for different purposes like cellular, television, military, emergency and satellite communication. The frequency spectrum used for cellular communication is getting over crowded with increasing number of subscribers and demand for high data rate text or video transmission, but the frequency spectrum in other wireless broadcast and communication is not utilised efficiently, example in Television broadcast band, some of frequency spectrum is vacant at some instant or for particular time. By FCC in 2002 reported that $70 \%$ of the spectrum at certain time and location is ideal, which shows that there is no scarcity of spectrum but underutilization of spectrum. The vacant spectrum could be used in cellular communication, means the spectrum is borrowed from Television Broadcast band to be used for cellular communication. A use of spectrum of licensed frequency bands when primary user is absent, will improve the efficiency of spectrum utilization [1].
By the mean of Cognitive Radio technology, the high data rate could be achieved for multimedia applications. Cognitive Radio covers the services such as speaker recognition, language identification then translation into another, text-tospeech conversion, speech-to-text conversion, noise suppression, and noise management. For military applications, Cognitive Radio will allow finding free channel automatically to communicate instead of manually setting to a particular radio channel. Another benefit, $\mathrm{CR}$ can resist the jamming in a particular area where radio signal jammers are used and some radio spectrum is restricted. In disaster and emergency conditions, due to over-crowding of the radio spectrum, collapses the communication system. In such circumstances, when radio spectrum is completely occupied and no direct link is available to the access point but through other CR terminals, access point is reachable by forming spontaneous network [23].

The remainder of this paper is organized as follows: in Section II, the most popular spectrum sensing technique Energy Detection is discussed along with the flow chart explaining the method used for the detection of signal. Section III describes the Non-Time Dispersive Propagation Model: Hata Propagation Model. In section IV the system model specifications, for simulation on precisely built Cognitive Radio Environment for Global Systems for Mobile Communication using Spectrum Engineering Advanced Monte Carlo Analysis Tool (SEAMCAT), has been discussed. Section V includes the Simulation Results from different NonTime Dispersive Fading regions as rural, sub-urban and urban area. Whereas Section VI, describes the impact of variations in number of secondary users on interference probability. In Section VII we draw our conclusions. Section VIII describes the Impact of present study on Cognitive radio Performance while Section IX shows the Future Scope for continuation of the research work on efficient Cognitive Radio Spectrum Sensing Techniques.

\section{ENERGy Detection SPECTRUM SENSING TeCHNIQUE}

The secondary users are required to sense and monitor the radio spectrum within their operating range to detect the frequency bands that are not occupied by primary users. In this section, we discuss the most popular spectrum sensing 
scheme, the energy detection. The energy detector employs a non-coherent detection technique, which does not require prior knowledge of pilot data. Energy detector spectrum sensing based approach, also known as radiometry or periodogram, is the most common way of spectrum sensing because of its low computational and implementation complexities. In addition, it is more generic as receivers do not need any knowledge on the primary user's signal. The energy of the received signal at secondary user is measured over an observation time window and compared with some threshold value to detect the presence of primary user over that particular channel. Mathematically, the energy detection method is a DFT based method to estimate power spectral density (PSD) and the energy of the input signal and compares it with a threshold energy value. The signal is said to be present at a particular frequency if the energy of the signal exceeds the threshold Energy level [3].Energy Detection in IEEE 802.22 WRAN system is possible using the two methods namely (i)Received Signal Strength Measurement(RSSI)(ii)Multi resolution Spectrum Sensing(MRSS).In RSSI approach, one can select the unoccupied channels using received signal strength by converting the energy in an interested band to the input signal strength whereas in MRSS approach, it senses the interested band in the analog domain using a wavelet transform using Fourier Transform. Since, it is performed in analog domain, so spectrum sensing can be reduced. Above cited Energy Detection technique has been followed as shown in Figure 1 below.

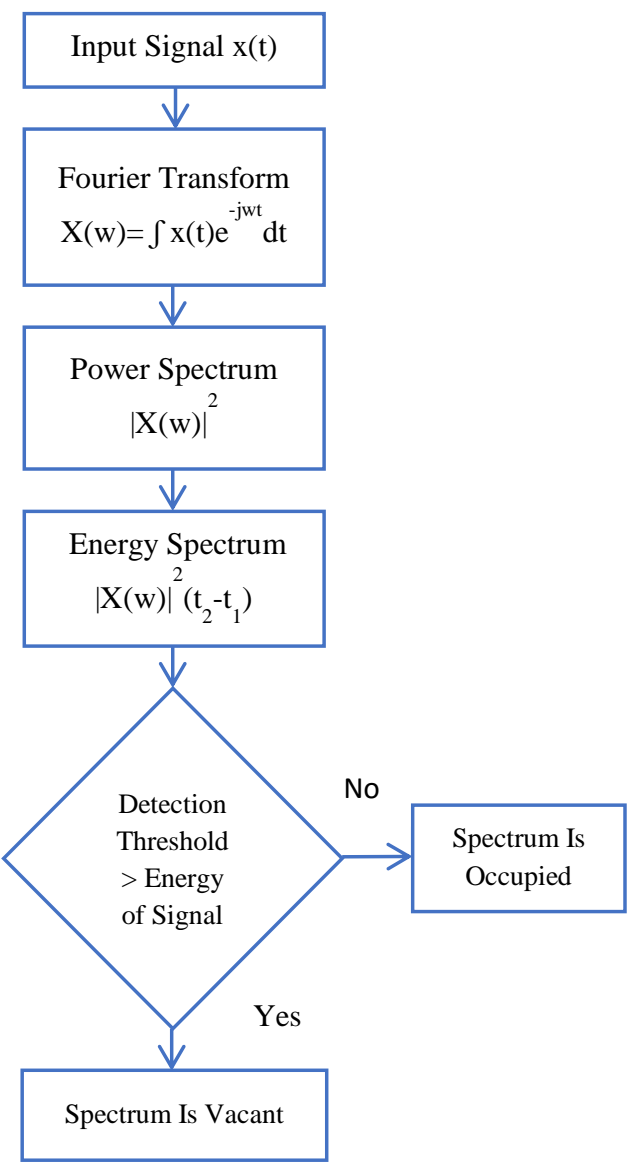

Fig. 1. Flowchart for Energy Detection Spectrum Sensing Technique

\section{NON-TIME DisPERSIVE PROPAGATION MODEL}

Wireless channel models are critical to both the analysis of interference to primary receivers in CR environment and the design of real-time methods to control such interference. An empirical model is based on data used to predict, not explain a system and are based on observations and measurements alone. It can be split into two subcategories, time dispersive and non-time dispersive. The time dispersive model provides the information about time dispersive characteristics of the channel like delay spread of the channel during multipath.

The Stanford University Interim (SUI) model is the perfect example of this type. The non-time dispersive provides the information about loss of signal strength in different propagation environments. Hata and ITU-R model are examples of non-time dispersive empirical model [4]. Radio propagation is also just as hard to predict as weather. Too many parameters have to be measured real-time, and too many details of terrain and obstacles have to be modelled to get accurate results. The extensive measurements of urban, suburban and rural radio environment losses, Okumura et al. published many empirical curves useful for cellular systems planning. These empirical curves reduced to a convenient set of formulas known as the Hata model. The basic formula for the median propagation loss given by Hata is: -

$$
\begin{array}{ll}
\text { fpropagation }(f, h 1, h 2, d, e n v)=L+T(G(\sigma)) \\
\text { Where } \mathrm{f}: \quad \text { frequency }(\mathrm{MHz}) \\
\mathrm{h}_{1}: \quad \text { transmitter antenna height, } \mathrm{m} \text {, above ground } \\
\mathrm{h}_{2}: \quad \text { receiver antenna height, } \mathrm{m}, \text { above ground } \\
\mathrm{d}: \quad \text { distance } \mathrm{b} / \mathrm{w} \text { transmitter and receiver }(\mathrm{km}) \\
\text { env: } \quad \text { general environment. } \\
\text { Symbols: } \mathrm{L}=\text { median propagation loss }(\mathrm{dB})
\end{array}
$$

\section{SYSTEM SPECIFICATIONS}

The virtual simulator has been chosen to test the impact of environmental fading over the working of Cognitive Radio's or White Space Devices using energy detection spectrum sensing technique, is Spectrum Engineering Advanced Monte Carlo Analysis Tool [5-6].

The SEAMCAT tool models a primary mobile station transmitter $\left(\mathrm{P}-\mathrm{MS}_{\mathrm{Tx}}\right)$ connected to a primary base station receiver $\left(\mathrm{P}-\mathrm{BS}_{\mathrm{Rx}}\right)$ which describes a primary user link and operating amongst a secondary mobile station transmitter (S$\mathrm{MS}_{\mathrm{Tx}}$ ) acting as a interferer to the primary user spectrum or channel as shown in the Figure2.

A secondary user belongs to either same system as the primary or different. The interferers are randomly distributed around the primary base station receiver in a manner decided by the user. It is common practice to use a uniform random distribution.

\section{A. Calculation of Interference Probability}

If

$$
\frac{C}{I_{\text {trail }}}>\frac{C}{I_{\text {target }}}
$$

Then there is no interference and event is considered good. 


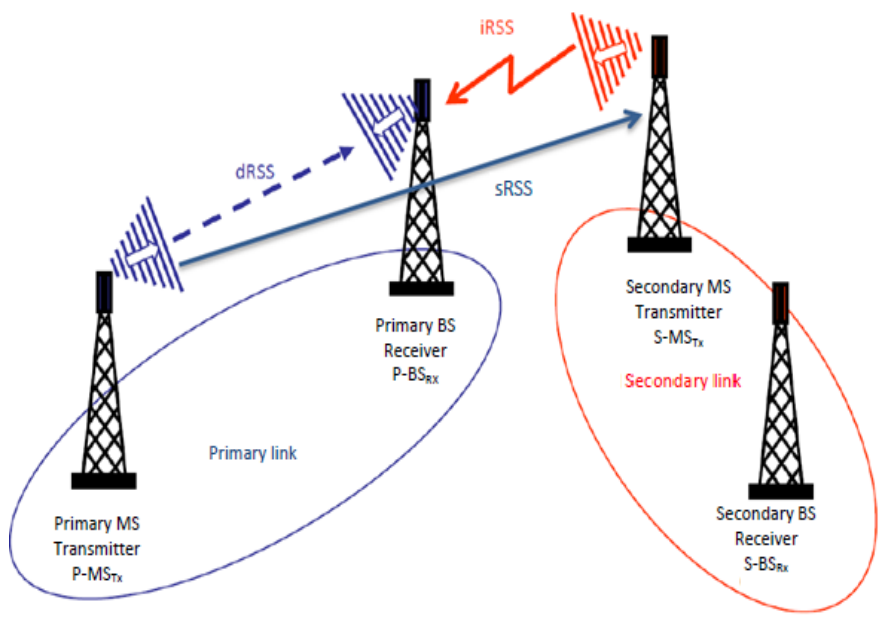

Fig. 2. Primary and secondary user modelling in SEAMCAT Where: dRSS desired Received Signal Strength; iRSS - interfering Received Signal Strength; sRSS - sensing Received Signal Strength

If

$$
\frac{C}{I_{\text {trail }}}<\frac{C}{I_{\text {target }}}
$$

Then interference exists.

The probability of interference is given by: -

$$
P_{\text {interference }}=1-\left(\frac{N_{\text {good }}}{N_{\text {all }}}\right)
$$

Where: $\mathrm{N}_{\text {all }}=$ total random events for particular simulation

$\mathrm{N}_{\text {good }}=$ good events i.e. no interference.

The sRSS(sensing Received Signal Strength) at the channel ' $m$ ' is calculated as follows.

$$
\begin{aligned}
& S R S S\left(f_{m}\right)=P_{P-M S T x}\left(f_{m}\right)+G_{P-M S T x \rightarrow S-M S T x} \\
& \quad+G_{S-M S T x \rightarrow P-M S T x}+L
\end{aligned}
$$

Where $\quad \mathrm{P}_{\mathrm{P}-\mathrm{MSTx}}$ : is the transmit power in $\mathrm{dBm}$

$\mathrm{f}_{\mathrm{m}}$ : $\quad$ is the frequency of the WSD.

$\mathrm{G}_{\mathrm{P}-\mathrm{MSTx} \rightarrow \mathrm{S}-\mathrm{MST} \mathrm{x}}$ : antenna gain in $\mathrm{dBi}$ of the $\mathrm{P}-\mathrm{MS}_{\mathrm{Tx}}$, in the $\mathrm{P}-\mathrm{MS}_{\mathrm{Tx}}$ to $\mathrm{S}-\mathrm{MS}_{\mathrm{Tx}}$ direction.

$\mathrm{G}_{\mathrm{S}-\mathrm{MST} \rightarrow \mathrm{P}-\mathrm{MSTx}}$ : antenna gain in $\mathrm{dBi}$ of the $\mathrm{S}-\mathrm{MS}_{\mathrm{Tx}}$ in the $\mathrm{S}-\mathrm{MS}_{\mathrm{Tx}}$ to $\mathrm{P}-\mathrm{MS}_{\mathrm{Tx}}$ direction.

$\mathrm{L}$ : $\quad$ is the path loss in $\mathrm{dB}$ between the $\mathrm{S}-\mathrm{MS}_{\mathrm{Tx}}$ and the P-MS $\mathrm{Mx}$.

The non-cooperative spectrum access technique is used with the energy detection spectrum sensing [7]. The study has been conducted for the Global System for Mobile Communication, considering two channels with channel spacing $200 \mathrm{kHz}$ to study the effect of detection threshold on interference at primary user receiver or victim receiver by single secondary user and to study the impact of increasing the number of secondary users or WSD on a primary user. Figure 3 shows the flowchart for the process of calculation of interference. The frequencies of the Primary Mobile Station Transmitter $\left(\mathrm{P}-\mathrm{MS}_{\mathrm{Tx}}\right)$ and Secondary Mobile Station Transmitter $\left(\mathrm{S}-\mathrm{MS}_{\mathrm{Tx}}\right)$ are same i.e. $890.1 \mathrm{MHz}$ and 890.3 $\mathrm{MHz}$ because the Secondary Mobile Station Transmitter (S$\mathrm{MS}_{\mathrm{Tx}}$ ) is a Cognitive Radio Device that will find the vacant channel in accordance with the detection threshold specified for it.The distance between primary mobile station transmitter
$\left(\mathrm{P}-\mathrm{MS}_{\mathrm{Tx}}\right)$ and primary base station receiver $\left(\mathrm{P}-\mathrm{BS}_{\mathrm{Rx}}\right)$ is fixed to 100 metres and the simulation radius of secondary mobile station transmitter $\left(\mathrm{S}-\mathrm{MS}_{\mathrm{Tx}}\right)$ for random distribution around $\mathrm{P}$ $\mathrm{BS}_{\mathrm{Rx}}$ is approximately 40 metres. The distance between secondary mobile station transmitter $\left(\mathrm{S}-\mathrm{MS}_{\mathrm{Tx}}\right)$ and secondary base station receiver $\left(\mathrm{S}-\mathrm{BS}_{\mathrm{Rx}}\right)$ is also fixed to 100 metres.

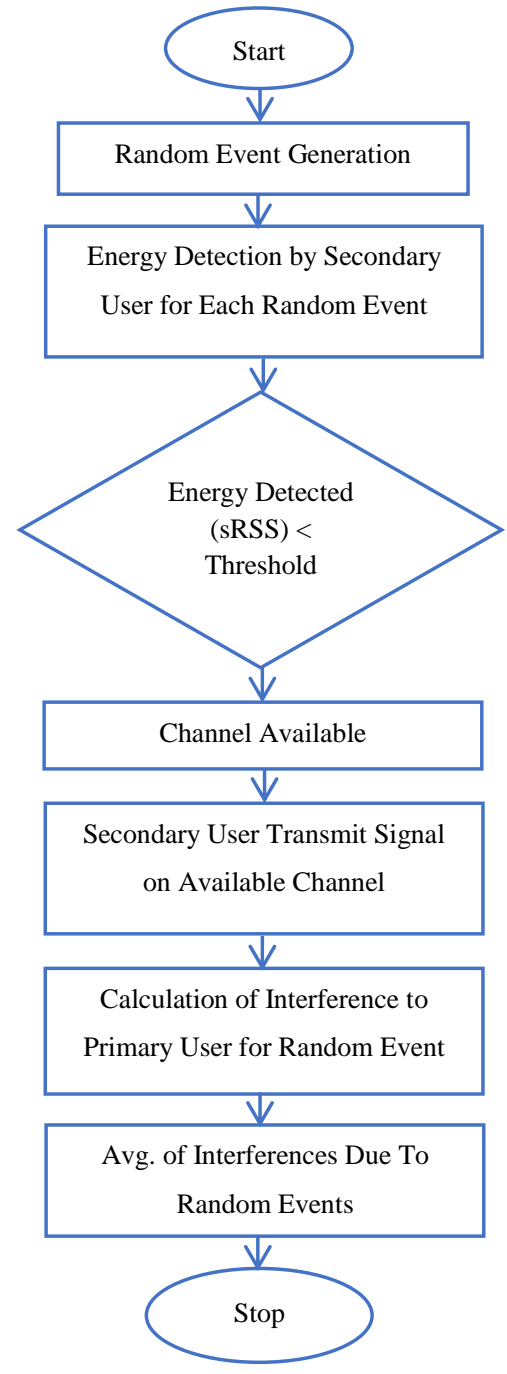

Fig. 3. Flow process of interference calculation

The antenna gain of mobile station transmitters and base station transmitters are $0 \mathrm{~dB}$ and $9 \mathrm{~dB}$ respectively. The antenna's pattern is omni-directional. The carrier to interference ratio $(\mathrm{C} / \mathrm{I})$ is considered $19 \mathrm{~dB}$ and sensitivity of receiver is $-104 \mathrm{dBm}$. From this, the noise floor is $-123 \mathrm{dBm}$ and the blocking mask of the $\mathrm{P}-\mathrm{BS}_{\mathrm{Rx}}$ receiver is constant that is $-23 \mathrm{dBm}$. Propagation model has been used for study, is Extended Hata model with variables in three different environments that are rural, sub-urban and urban areas. In model, the location of transmitter is also changed either indoor or outdoor and loss of walls is taken into consideration for study which is $10 \mathrm{~dB}$ for signal transmission from indoor to outdoor. The base station receiver is fixed outdoor.The propagation model specifications are kept same for each propagation link as the primary mobile station transmitter $(\mathrm{P}$ $\left.\mathrm{MS}_{\mathrm{Tx}}\right)$ to the primary base station receiver $\left(\mathrm{P}-\mathrm{BS}_{\mathrm{Rx}}\right)$, secondary 
mobile station transmitter $\left(\mathrm{S}-\mathrm{MS}_{\mathrm{Tx}}\right)$ to the primary base station receiver $\left(\mathrm{P}-\mathrm{BS}_{\mathrm{Rx}}\right)$ and the primary mobile station transmitter $\left(\mathrm{P}-\mathrm{MS}_{\mathrm{Tx}}\right)$ to the primary base station receiver $\left(\mathrm{P}-\mathrm{BS}_{\mathrm{Rx}}\right)$ [89].The power transmitted by both $\mathrm{P}-\mathrm{MS}_{\mathrm{Tx}}$ and $\mathrm{S}-\mathrm{MS}_{\mathrm{Tx}}$ is 33 $\mathrm{dBm}$ and the emission mask of transmitters is considered as per the recommendation of the European Telecommunications Standards Institute (ETSI).The detection threshold for energy detection is considered with different constant values in $\mathrm{dBm}$ for different studies. The probability of failure or false alarm is considered $10 \%$ for different propagation environments from prospective of comparative analysis (It could be different for different areas and may be much lower than 10\%)and the reception bandwidth of secondary mobile station is $200 \mathrm{kHz}$.

\section{Simulation Results AND Discussion}

In this study, impact of changing a detection threshold of the White Space Devices on interference at primary user is studied for two picocell communication systems to share two primary channels. The interference has been compared with the detection threshold of energy detection spectrum sensing in terms of probability of unwanted interference occurrence. The comparison has been done for inter wireless radio environments for both indoor and outdoor mobile station transmitters as primary and secondary users as shown in Figure 4 and Figure 5. Then the comparison has been done for intra wireless radio environment such as rural area with indoor and outdoor mobile station transmitters as shown in figure 6, 7 $\& 8$. The probability of interference is only accounted for the unwanted signal because the interference due to blocking is very low and negligible. The probability of interference has been increasing and attaining maximum value at different detection threshold values for different wireless radio environments for outdoor primary and secondary user transmitters. For rural area, interference arises at higher detection threshold value than sub-urban area and for suburban; it arises at higher detection threshold value than urban area. In rural area, interference probability arises as detection threshold value increases above $-90 \mathrm{dBm}$ and becomes constant and highest above $-10 \mathrm{dBm}$; in sub-urban area, interference probability arises as detection threshold value increases above $-110 \mathrm{dBm}$ and becomes constant and highest above $-30 \mathrm{dBm}$ and in urban area, interference probability arises as detection threshold value increases steeply above $110 \mathrm{dBm}$ and becomes constant and highest above $-50 \mathrm{dBm}$ as shown in figure 4.

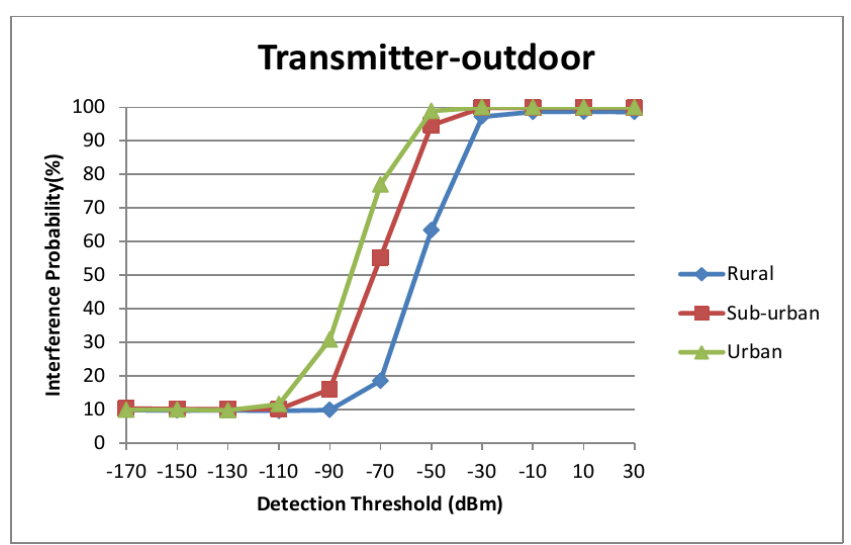

Fig. 4. Inter-Wireless Radio Environment with Outdoor Transmitters (DT)

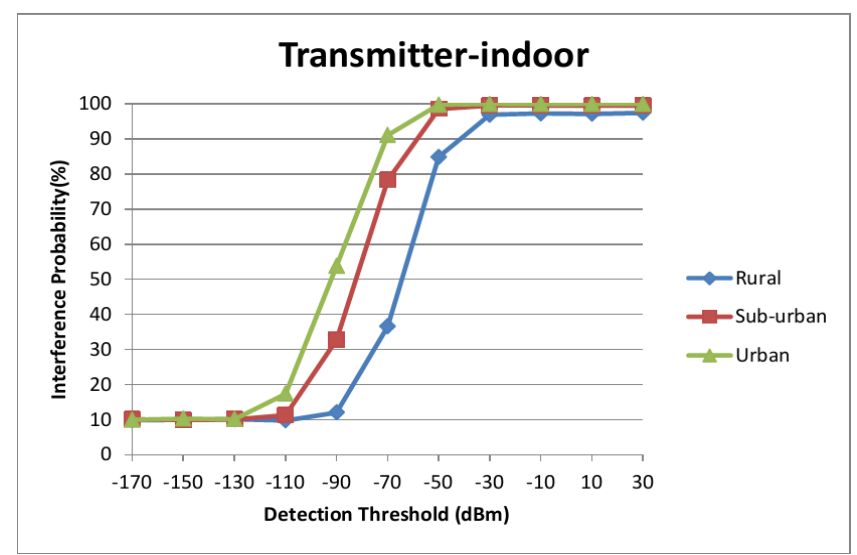

Fig. 5. Inter-Wireless Radio Environment with Outdoor Transmitters (DT)

In rural area, interference probability arises as detection threshold value increases above $-100 \mathrm{dBm}$ and becomes constant and highest above $-30 \mathrm{dBm}$; in sub-urban area, interference probability arises as detection threshold value increases steeply above $-110 \mathrm{dBm}$ and becomes constant and highest above $-50 \mathrm{dBm}$ and in urban area, interference probability arises as detection threshold value increases above $-130 \mathrm{dBm}$ and becomes constant and highest above $-60 \mathrm{dBm}$ as presented in figure 5.The probability of interference increases when the mobile station primary and secondary users are moved from outdoor to indoor environment at particular detection threshold. For intra-wireless radio environment: rural area, the interference probability arises at $100 \mathrm{dBm}$ when the transmitters are indoor and arises at -90 $\mathrm{dBm}$ when the transmitters are outdoor as shown in fig 6.

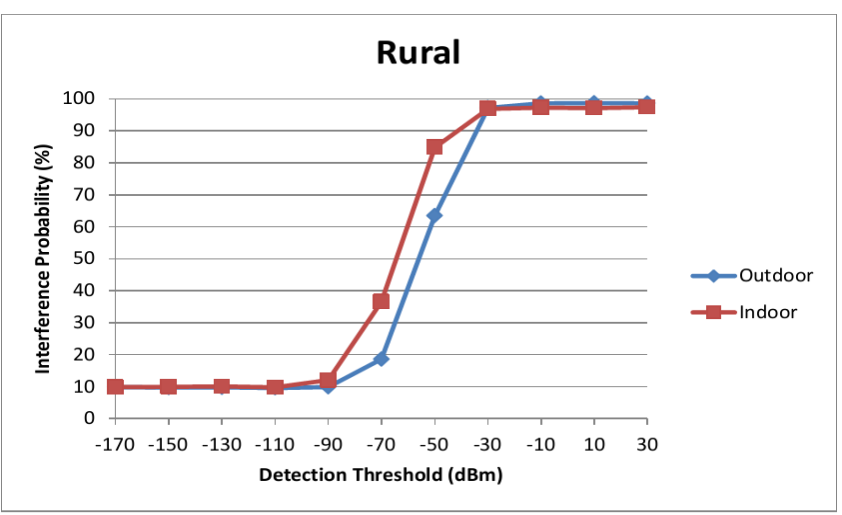

Fig. 6. Intra-Wireless Radio Environment - Rural Area (DT)

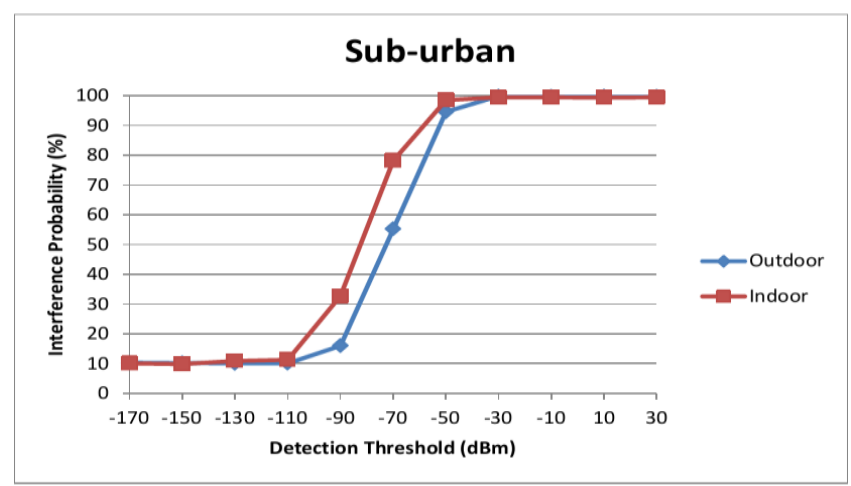

Fig. 7. Intra-Wireless Radio Environment - Sub-urban Area (DT) 
In intra -wireless radio environment for both sub-urban and urban areas, the detection threshold for indoor transmitters is required less than the detection threshold for outdoor transmitters as predicted in Figure 7 and Figure 8 respectively.

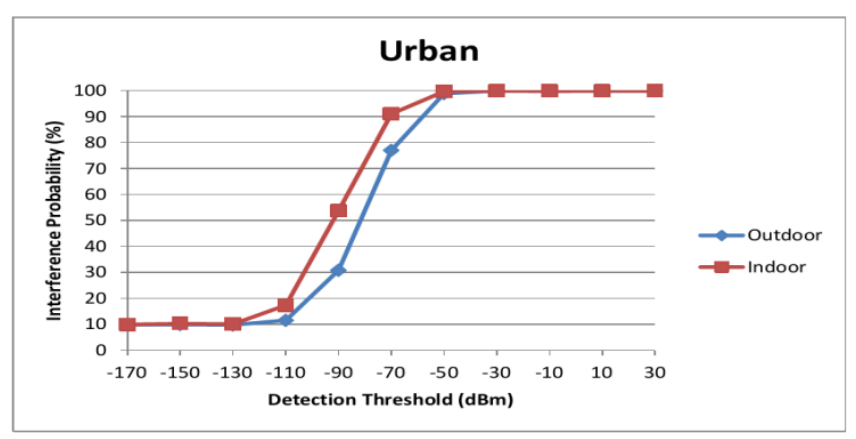

Fig. 8. Intra-Wireless Radio Environment - Urban Area (DT)

The interference probability arises as mobile station users moved from outdoor to indoor environment at particular detection threshold value due to the increase in the path loss and decrease in Sensing Received Signal Strength; which makes more randomly generated samples to fall under lower value of detection threshold.

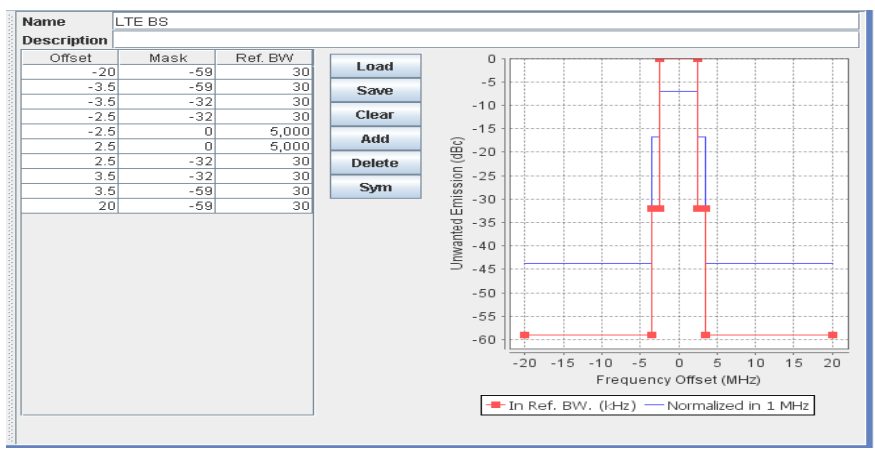

Fig. 9. shows the Spectral Emission Mask for LTE-BS

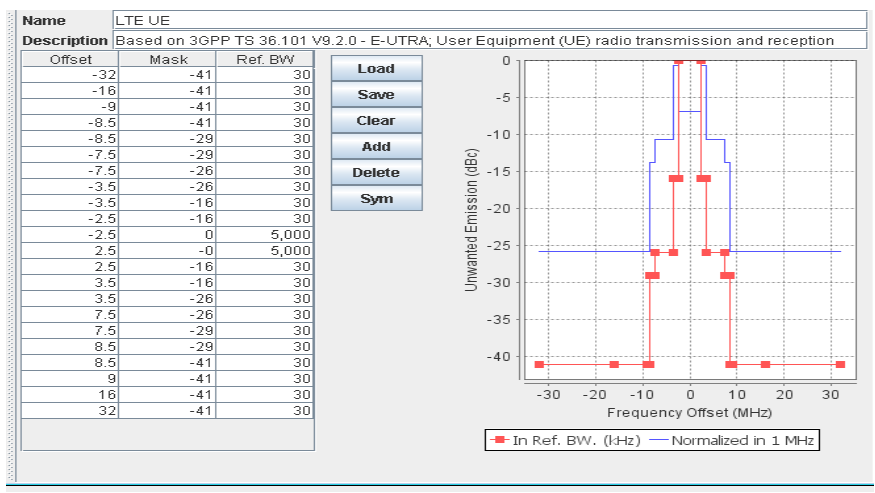

Fig. 10. shows the Spectral Emission Mask for LTE-UE

Figures 9 and 10 show the Effective Isotropic Radiated Power [EIRP] in block mask using SEAMCAT which inputs the In-block CR emission limit as a triplet offset, Mask, Ref.BW] where Offset in Mhz is equivalent to the channel spacing, Mask in $\mathrm{dBm}$ is the In-block CR EIRP max limit and Ref BW is the bandwidth of the primary base station receiver.
TABLE I. ShOWS SRSS VALUES WITH OUTDOOR TRANSMITTERS

\begin{tabular}{|l|l|l|l|}
\hline Areas & Mean & Min & Max \\
\hline Rural & -53.75 & -101.88 & -7.80 \\
\hline Sub Urban & -70.38 & -125.06 & -23.25 \\
\hline Urban & -79.50 & -129.41 & -29.64 \\
\hline
\end{tabular}

TABLE II. SHOWS SRSS VALUES WITH INDOOR TRANSMITTERS

\begin{tabular}{|l|l|l|l|}
\hline Areas & Mean & Min & Max \\
\hline Rural & -63.55 & -117.03 & -12.33 \\
\hline Sub Urban & -80.49 & -138.99 & -30.02 \\
\hline Urban & -89.37 & -150.12 & -32.62 \\
\hline
\end{tabular}

The minimum and maximum sRSS values or cumulative probabilities of sRSS values could differ little due to the generation of random samples for every simulation but a mean value is approximately same.

\section{VARIATIONS IN INTERFERENCE PROBABILITY DUE TO CHANGE IN NUMBER OF SECONDARY USERS}

In this study, the impact of increasing the number of mobile station secondary users over the primary link has been studied in terms of interference probability within different radio environment[10-11].The detection threshold is assumed $-100 \mathrm{dBm}$ that is average value of all detection threshold values for inter wireless radio environment. The interference probability for each number of secondary users for indoor transmitter's in sub urban area results in same interference probability as for outdoor transmitters in urban area due to the probability of random generation events of sensing Received Signal Strength under detection threshold being same for both. Wireless channel models are critical to both the analysis of interference to primary receivers in CR environment and the design of real time methods to control such interference[12$13]$.

\section{CONCLUSION}

The difference in the sensing Received Signal Strength, the interference experienced by the primary link base station receiver is also different with changing the values of detection threshold in different wireless radio environment. At highest detection threshold, the interference experienced by the base station receiver has achieved its highest value for each radio environment and the interference occurs by secondary mobile station transmitter using both adjacent and co-channel in equal proportion of randomly generated events. As the detection threshold decreases, then the presence of primary user is detected more precisely and the interference is reduced as the secondary user is not allowed to transmit over the frequency channels used by the primary user. The cognitive radio should be programmed for lesser detection threshold value for the urban area than other areas to achieve minimum interference probability at primary users. Cognitive radio is a futuristic technology that will utilize the spectrum efficiently and globalize the radio environment for different band users which use different techniques for transmission.

\section{IMPACT OF STUDY}

The study will be useful in radio environment which is opportunistic, adaptive and intelligent under different strategic 
conditions. This study will provide base for maximum capacity or spectrum utilization under different wireless fading environment which will be helpful in the design of Intelligent Wireless Communication System under the constraints of Interference. The study has its impact on design and development of cognitive radio system under ubiquitous pervasive environment [14-18].

\section{FUTURE SCOPE}

It is well-known that energy detector's performance is susceptible to uncertainty in noise power under such cases alternate detection schemes such as cyclic feature detection, Wavelet based detection and Filter bank multicarrier approach or power control scheme may be employed. The Performance analysis of spectrum sensing in this scenario is the subject of our current research.

Further study will be carried out by accounting other wireless radio environment parameters such as refractivity, troposphere scatter, etc using propagation models like ITU-R P.452-14 over the performance of Cognitive radio using energy detection spectrum sensing. The effect of small scale fading can be accounted for different wireless radio environment. The impact of variable distance can be studied using cooperative approach in future by modeling different wireless radio propagation environments for Cognitive radio as the number of secondary users is more than one. The interference effect could be studied using power control feature for secondary users in different wireless radio environment.

\section{ACKNOWLEDGEMENT}

The First Author is thankful to the Research Promotion Cell,Panjab University Chandigarh for providing the publication grant under Improvement of Education Budget Head.

\section{REFERENCES}

[1] J. Mitola III, "Software radios: Survey, critical evaluation and future directions," IEEEAerospace and Electronic Systems Magazine, vol. 8, pp. 25-36, Apr. 1993.

[2] J. Mitola III, "Cognitive radio for flexible mobile multimedia communications," in Proceedings of the IEEE International Workshop on Mobile Multimedia Communications, SanDiego, CA, USA, vol. 1, pp. 3-10, Nov. 1999.

[3] S. Haykin, "Cognitive radio: Brain-empowered wireless communications," IEEE Journal on Selected Areas in Communications, vol. 23, pp. 201-220, Feb. 2005.
[4] V.S. Abhayawardhana, I.J. Wassel, D. Crosby, M.P. Sellers, M.G. Brown, "Comparison of empirical propagation path loss models for fixed wireless access systems," 61th IEEE Technology Conference, Stockholm, pp. 73-77, 2005.

[5] Spectrum Engineering Advanced Monte Carlo Analysis Tool, online: http://www.ero.dk/seamcat.

[6] Spectrum Sensing capabilities in SEAMCAT, by Stella Lyubchenko, Jean-Philippe Kermoal, Marc Le Devendec, Int. Symposium on Electromagnetic Compatibility, York, UK, 2011, pp. 658-663.

[7] "Non-Cooperative Spectrum Access - The Dedicated vs. Free Spectrum Choice," by K. Jagannathan, I. Menache, E. Modiano, G. Zussman, in Areas in Communications, IEEE Journal on , vol.30, no.11, pp.2251,2261, December 2012.

[8] "Modeling and Validation of Channel Idleness and Spectrum Availability for Cognitive Networks," by Ghosh, C.; Roy, S.; Rao, M. B.,Selected Areas in Communications, IEEE Journal on , vol.30, no.10, pp.2029,2039, November 2012.

[9] Digital cellular telecommunications system-Radio transmission and reception (GSM 05.05), by European Telecommunications Standards Institute 1996, online: www.etsi.org/deliver/etsi_gts/05/0505/05.../gsmts_0505v050000p.pdf.

[10] Fadel F.Digham,Mohd Slim Alouini ,’On the Energy Detection of Unknown Signals over Fading Channels,"Proc.IEEE International Conference,2003.pp.3575-3579.

[11] Amir Ghasemi and Elvino S Sousa,"Fundamental Limits of Spectrum Sharing in Fading Environments,"IEEE Transactions on Wireless Communications,Vol.6,No.2,Feb 2007,pp.649-658.

[12] Natasha Devroye,Patrick Mitran,"Achievable Rates in Cognitive Radio Channels,"IEEE Transactions on Wireless Communications,Vol.6,No.2,Feb 2007,pp.649-658.

[13] Hongiian Sun et al, "Computationally Tractable Model of Energy Detection Performance over Slow Fading Channels,"IEEE Communication Letters,Vol.14,No.10,pp.924-926.

[14] JaisukhPaul Singh, A. S. Kang et al "Cooperative Sensing for Cognitive Radio: A Powerful Access Method for Shadowing Environment, SPRINGER-Journal of Wireless Personal Communications,2014.Vol.80,Pgs15.

[15] A.S.Kang, Renu Vig, "Comparatative Performance Analysis of FBMC Prototype Filter Under Strategic Conditions,"European Journal of Scientific Research,Vol.125,No.3,October,2014.pp.362-369.

[16] A.S.Kang, Jaisukh Paul Singh et al, "Cooperative Fusion Sensing Technique for Cognitive Radio for Efficient Detection Method for Shadowing Environment,"Proc.Wilkes International Conference for Computing Sciences,ISBN:978-935107-172-3,Elseveir,2013,pp.70-79.

[17] A.S.Kang and Renu Vig, "Computer Aided BER Performance Analysis of FBMC Cognitive Radio for Physical Layer under the Effect of Binary Symmetric Radio Fading Channel,"Wireless Personal Communication" (C) Springer Science+Business Media New York 2015,Vol.81(2)pages.16.

[18] A.S.Kang, Jaisukh Paul Singh et al, "Cognitive Radio: State of Research Domain in Next Generation Wireless Networks-A Critical Analysis, "International Journal of Computer Applications(0975-8887)Vol.74No.10,July2013,pp.1-9. 